\title{
Tracking, alignment, and b-tagging performance and prospects in the ATLAS experiment
}

\author{
Nicholas Styles; on behalf of the ATLAS Collaboration \\ Deutsches Elektronen-Synchrotron (DESY) \\ E-mail: nicholas.stylesedesy.de
}

\begin{abstract}
Procedures employed for the reconstruction of charged particle tracks used by the ATLAS experiment are outlined, and their performance in recent LHC Run 2 data is discussed, with focus on aspects such as the stability with respect to time and instantaneous luminosity, and performance within high- $p_{T}$ jets. The track-based alignment of the ATLAS Inner Detector is introduced, and its importance to track reconstruction is demonstrated. The current level of understanding of the deformations of the detector which affect the determination of the track parameter is shown, and the planned future improvements are also outlined. Methods devised to distinguish $b-$ and $c$-quark jets from light-flavour jets are introduced. Techniques to extract and calibrate the performance of these methods on data and Monte Carlo are examined, and their results compared. Finally, forthcoming improvements in these areas are highlighted.
\end{abstract}

Sixth Annual Conference on Large Hadron Collider Physics (LHCP2018)

4-9 June 2018

Bologna, Italy

\footnotetext{
* Speaker.
} 


\section{Introduction}

The precise identification and determination of charged particle trajectories (or 'Tracks') in the ATLAS Inner Detector (ID) is a vital part of event reconstruction at the ATLAS experiment [1], as virtually all final-state objects used in analyses rely on tracks to some degree. A particularly good example of this is $b$-tagging, which uses tracks and their properties to efficiently and accurately identify jets initiated by $b$-quarks (and $c$-quarks). Such $b$-jets are a common final state object (either as a direct requirement or as a veto) in analyses across the full spectrum of the ATLAS physics program. The performance of the $b$-tagging algorithms directly impacts the sensitivity of these analyses, and so providing excellent and well-understood tracking and $b$-tagging performance is of vital importance. One area that has to be particularly well understood to achieve the necessary levels of performance is the track-based alignment of the Inner Detector, where techniques have been refined to provide very precise information on the positions and movements of sensitive detector elements.

\section{Track Reconstruction}

The ATLAS Inner Detector comprises three different technologies which are used to reconstruct tracks: silicon pixels (Pixel detector), silicon microstrips (Semiconductor Tracker, SCT), and gaseous straw tubes (Transition Radiation Tracker, TRT). Reconstructed tracks are required to have at least 7 silicon (Pixel $+\mathrm{SCT}$ ) measurements (hits) on tracks, and a $p_{T}>500 \mathrm{MeV}$, as well as additional criteria on properties such as the number of hits shared with other tracks, and the number of missing hits where a hit would be expected (holes). The efficiency for reconstructing tracks in a typical minimum bias collision event is around $85 \%$ in the central barrel, decreasing to around $75 \%$ at the extremes of the acceptance in the forward endcaps [2]. For certain applications additional track requirements are applied, e.g. to reduce the rate of tracks from secondary interactions. One such case is in the use of tracking counting as an estimate of instantaneous luminosity, where the stability of the selection efficiency is crucial. Figure 1 shows selection efficiency in $Z \rightarrow \mu \mu$ events as a function of time (a) and instantaneous luminosity (b). A new track selection (2017 track sel.)

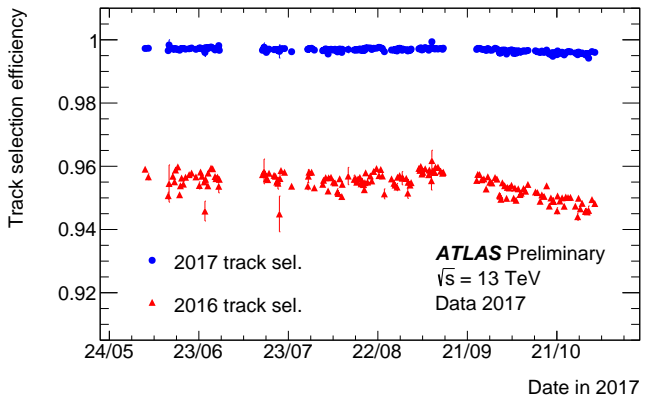

(a)

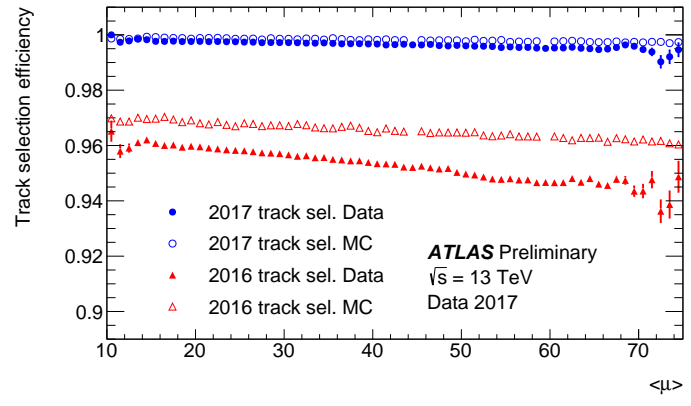

(b)

Figure 1: Track selection efficiency in $Z \rightarrow \mu \mu 2017$ as a function of time (a), and of instantaneous luminosity $(<\mu>)$ comparing data and Monte Carlo, for two different track selections [3].

was developed in order to improve stability with respect to what was used previously (2016 track 
sel.), through loosening the criteria on holes, and focusing on tracks in the ID barrel region [3]. As shown in Figure 1(b), this also improves agreement between data and Monte Carlo significantly.

Another application with specific needs and challenges, and of particular relevance to $b$ tagging, is the reconstruction of track within jets. Within high- $p_{T}$, highly collimated jets, the track density is very high and a high rate of hit sharing occurs, which can cause issues for efficient reconstruction (although specific reconstruction optimizations have been made to mitigate this effect [4]). This can be seen in Figure 2(a), which shows $r_{\text {track }}$, an observable sensitive to track reconstruction efficiency which is built from the ratio of $\Sigma p_{T}^{\text {track }}$ to jet $p_{T}$, as a function of jet $p_{T}$ [5]. The performance of track reconstruction within jets is very stable even for very high $p_{T}$ jets, as seen in Figure 2(b) which shows the ratio of $\Sigma p_{T}^{\text {track }}$ to jet $p_{T}$ as a function of time within 2016.

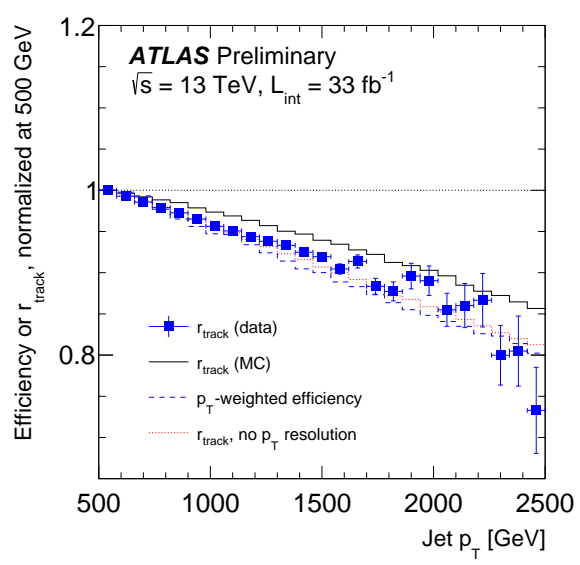

(a)

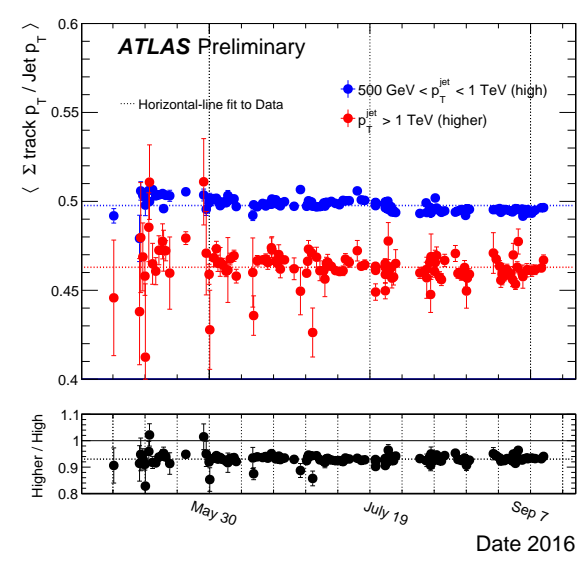

(b)

Figure 2: $r_{\text {track }}$ as a function of jet $p_{T}$ in data and Monte Carlo (a) [5], and the ratio of $\Sigma p_{T}^{\text {track }}$ to jet $p_{T}$ as a function of time within 2016 for two different jet $p_{T}$ ranges (b), with the ratio between the results in the two ranges in the bottom panel.

\section{Inner Detector Alignment}

Alignment of the ID is performed by using a track-based procedure, which iteratively minimizes the $\chi^{2}$ of the hit-to-track residuals at three levels of granularity, starting from the largest detector structures and ending with the individual sensors. Alignment at the highest-granularity level is performed approximately every 10 minutes during data-taking, to account for short timescale detector movements, such as the temperature-dependent bowing of the innermost pixel barrel layer (Insertable B-Layer, IBL) [7]. While achieving excellent alignment precision overall, this initial 'prompt' alignment cannot account for specific detector deformations under which the track $\chi^{2}$ is invariant. These so-called weak modes can bias various track parameters, and can only be controlled for once a statistically sufficient data set has been collected, by looking at external constraints such as the reconstructed $Z$ boson mass, and the ratio of momentum to calorimeter energy. The impact of re-aligning using these constraints is shown in Figure 3, which compares the level of charge-dependent momentum (sagitta) bias in the 'Prompt' (a) and 'Reprocessed' (b) alignment of 
2016 data, and displays the very low level of residual misalignments present in the final data used for analyses [8]. Work is ongoing to constrain and correct for further weak mode deformations that

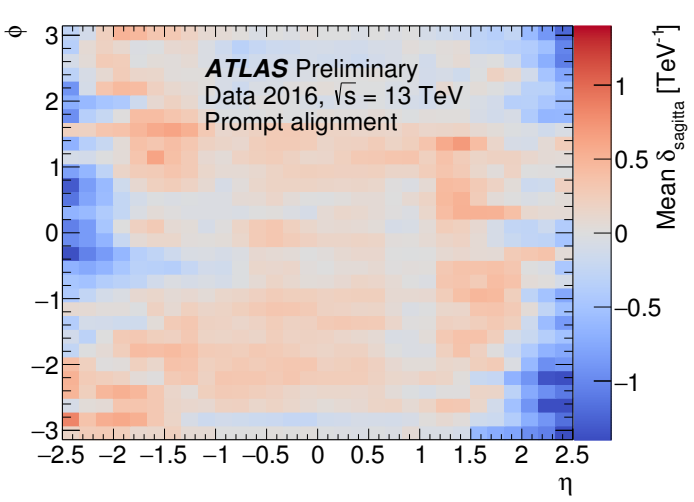

(a)

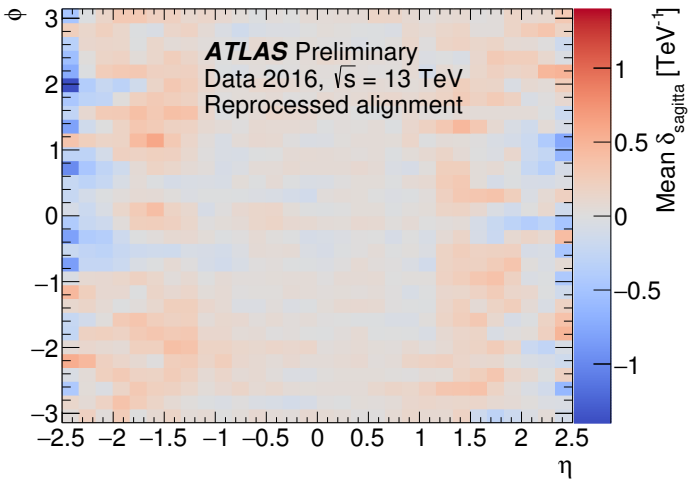

(b)

Figure 3: Measured residual sagitta bias as a function of track $\eta$ and $\phi$ in Prompt (a) and Reprocessed (b) 2016 data [8].

are not yet accounted for. An example of this is evidence of an effect consistent with a $\sim 250 \mu \mathrm{m}$ expansion of the SCT outer barrel layer [9]. The impact of such a radial expansion on a measured track is shown in Figures 4(a) and 4(b). The consistency of the observed effect across different momentum scales is shown in Figure 4(c).

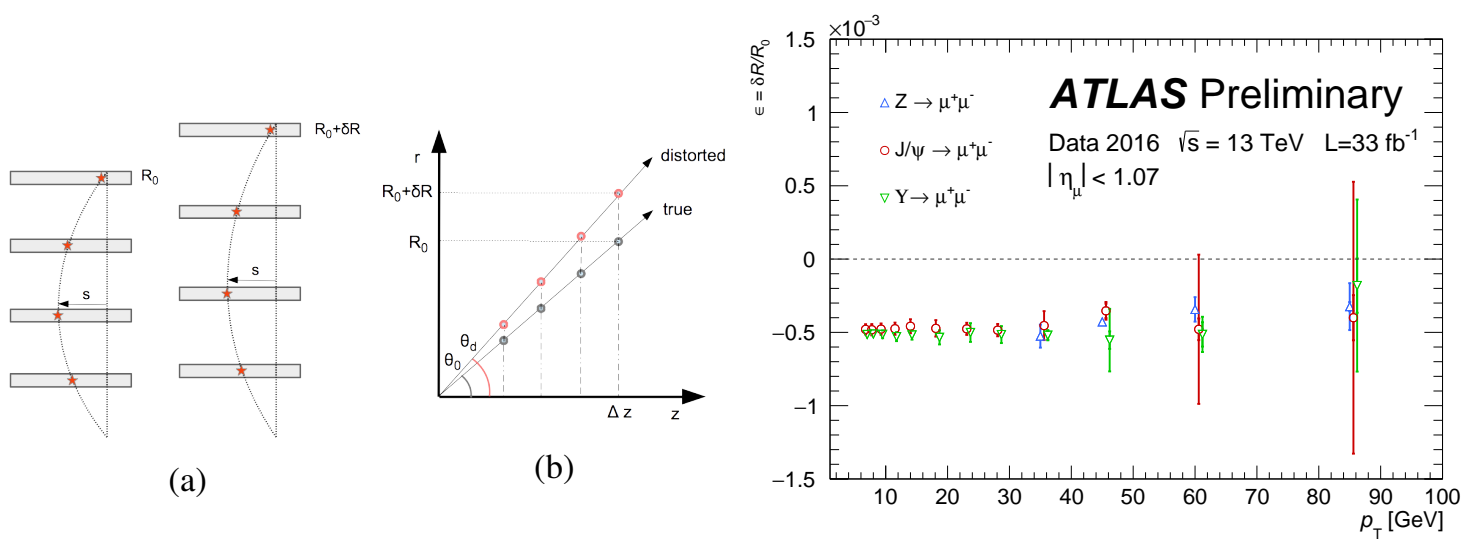

(c)

Figure 4: The impact of a radial expansion on the sagitta (a) and polar angle (b) of a track, and the measured radial distortion coefficient in 2016 data as a function of track $p_{T}$ (c) [9].

\section{4. $b$-tagging}

The identification of $b$-jets relies on several properties of $b$-quark decays, such as the track impact parameters, secondary decay vertices, and the decay topology which are exploited by several 'low-level' tagging algorithms. The output of these are combined into the final 'high-level' taggers, 
such as the MV2c10 algorithm which uses a Boosted Decision Tree to combine the low level information, which are used in analyses. A range of working points are provided, in order to give an appropriate balance of tagging efficiency to mistag rate for the analysis in question. The $b$-tagging must be carefully calibrated to account for any differences between the performance on data and Monte Carlo, and a variety of methods are used for this. Figure 5(a) shows the $b$-tagging efficiency extracted for a single working point (70\% efficiency) from a high purity dileptonic $t \bar{t}$ sample in data and Monte Carlo, using the so-called 'Combinatorial Likelihood' method [10]. This method uses per-event jet correlations to construct a likelihood function describing the $p_{T}$ and MV2c10 outputs of the jets in the event. The data to Monte Carlo scale factors are shown in Figure 5(b), including statistical and systematic uncertainties. The largest uncertainties come from the Monte Carlo generator modelling of the $t \bar{t}$ process. The scale factors extracted are generally close to unity for all working points, and have uncertainties varying from $2 \%$ to $12 \%$ depending on working point and jet $p_{T}$ range.

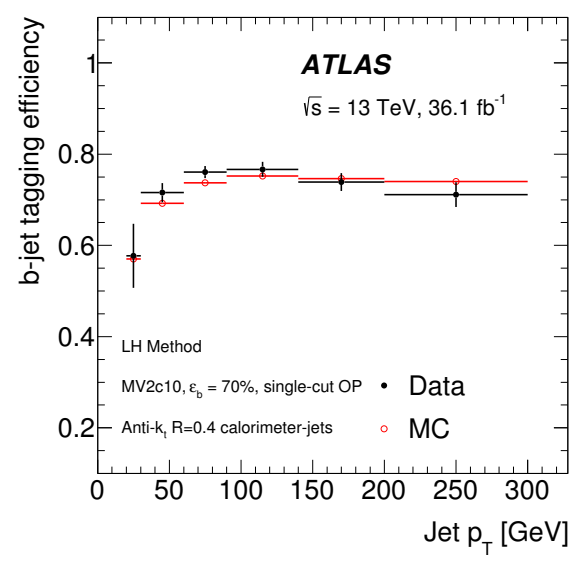

(a)

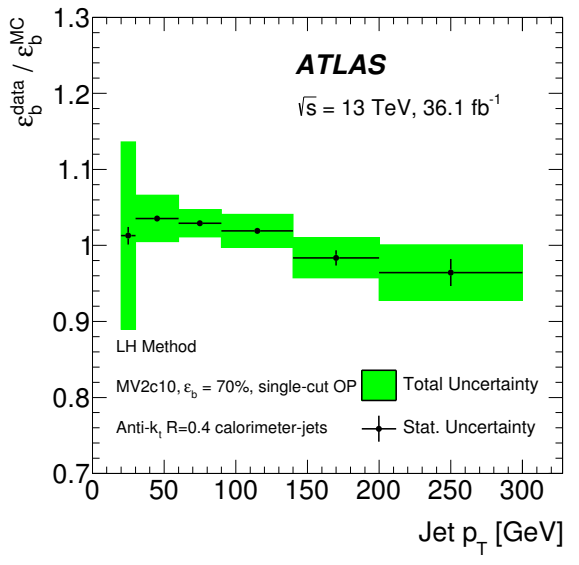

(b)

Figure 5: $b$-tagging efficiency as a function of jet $p_{T}$ for the $70 \%$ efficiency working point extracted via the Combinatorial Likelihood method in dileptonic $t \bar{t}$ data and Monte Carlo (a), and the data/Monte Carlo scale factors extracted from this including statistical and systematic uncertainties (b) [10].

Calibrations of the mistag rates, i.e. the rate at which light jets are classified as $b$-jets are also highly important. These mistag rates vary from $0.1 \%$ to $16 \%$ depending on the efficiency working point and kinematics. One method of calibrating the mistag rate performed uses dedicated studies of various tracking parameters and the differences observed in them between data and Monte Carlo, and adjusting the simulated tracks according to these differences to make it closely resemble data [11]. Of particular importance for $b$-tagging is the Impact Parameter, and the results of studies used to extract the Impact Parameter resolution, through an iterative procedure removing the contribution from the resolution on the primary vertex position, are shown in Figure 6. After applying the data/Monte Carlo differences shown in Figure 6(b) to the original Monte Carlo, along with other adjustments, mistag scale factors in the range of around 1.5 to 3 are derived, in agreement with those measured in other methods. 


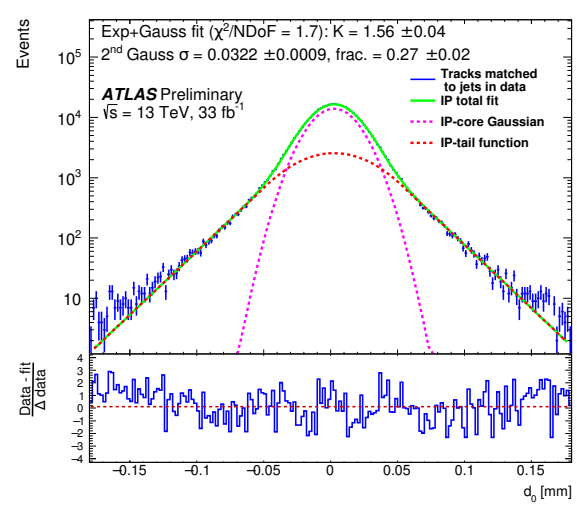

(a)

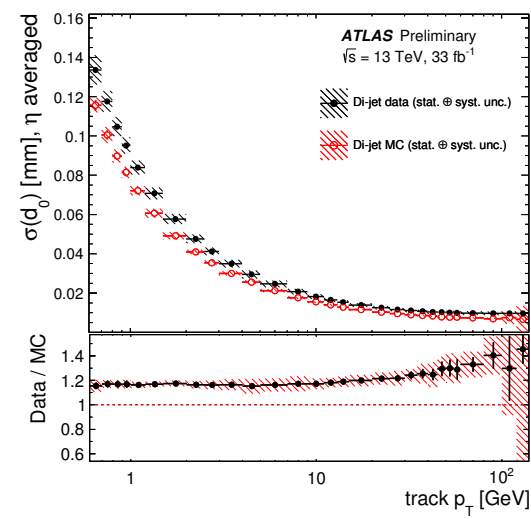

(b)

Figure 6: Fit of the core and tail resolution contribution to the track $d_{0}$ distribution after removal of the primary vertex resolution in a single bin with $13<p_{T}<15 \mathrm{GeV}$ and $0.75<\eta<1.0$ bin for Di-jet data (a), and the $d_{0}$ resolution as function of track $p_{T}$ in Di-jet data and Monte Carlo (b). [11]

For several applications it is also important to understand the rate of $c$-jets being tagged as $b$-jets, and this has been studied in single-lepton $t \bar{t}$ events in which a kinematic likelihood fit is performed to assign jets to the $t \bar{t}$ partonic final state. After requiring that both jets assigned to the $b$-jets from the $t$-quark decays are $b$-tagged, a likelihood fit is performed to extract the rate of tagged $c$-jets [12]. Figure 7(a) shows the agreement between data and Monte Carlo in the leading jet $p_{T}$ after the likelihood fit is performed, and Figure 7(b) shows the $c$-jet mistag rate scale factors extracted for various working points, including statistical and systematic uncertainties. The largest systematics are the $t \bar{t}$ Monte Carlo generator modeling, and the input data/Monte Carlo scale factors used for $b$ - and light jets.

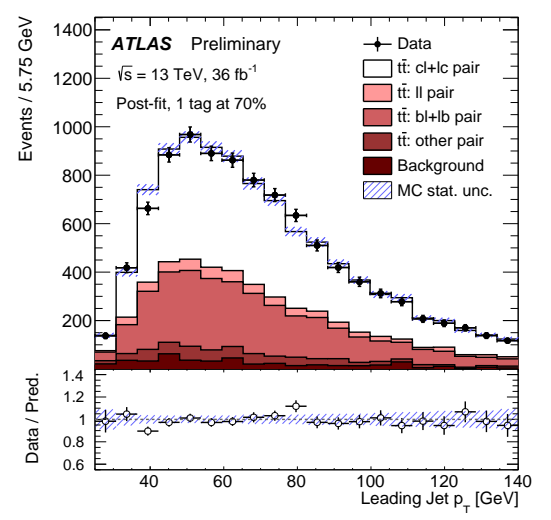

(a)

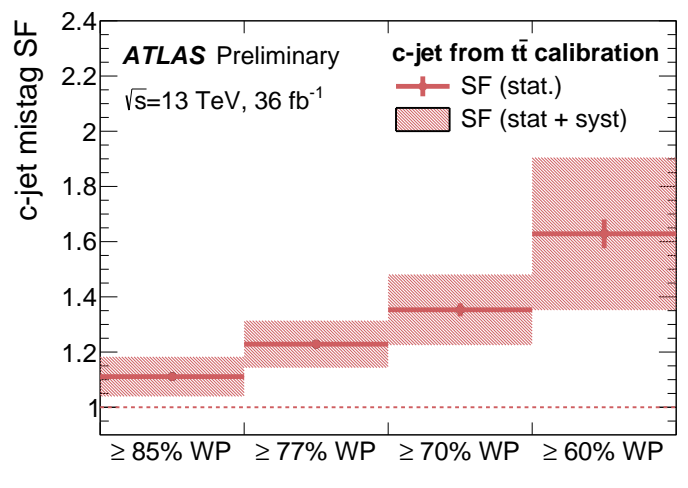

(b)

Figure 7: Distribution of the leading jet $p_{T}$ in single-lepton $t \bar{t}$ events after the kinematic likelihood fit and requiring one jet assigned to the $W$ boson decay to be $b$-tagged (a), and the extracted $c$-jet mistag rate scale factors for various efficiency working points, including statistical and systematic uncertainties (b) [12]. 


\section{Conclusions and Prospects}

The ATLAS experiment has achieved excellent performance up to now in the areas of tracking, alignment, and $b$-tagging. In-depth studies allow this performance to be very well understood and modelled. Nevertheless, there are numerous new developments planned for the future: updated detector descriptions in simulation will improve data to Monte Carlo agreement; in-depth studies of the radial alignment distortions will continue; new $b$-tagging algorithms using deep-learning techniques to combine low-level tagger information (in addition to improvements and additions to the low-level taggers themselves) are currently being commissioned [13]. Such improvements will allow ATLAS to continue increasing its physics reach toward the end of Run 2 and beyond.

\section{References}

[1] ATLAS Collaboration, The ATLAS experiment at the CERN large hadron collider, J. Nucl. Sci. Technol. 3, S08003 (2008).

[2] ATLAS Collaboration, Early Inner Detector Tracking Performance in the 2015 data at $\sqrt{s}=13 \mathrm{TeV}$, ATL-PHYS-PUB-2015-051 (cdsweb.cern.ch/record/2110140).

[3] ATLAS Collaboration, Track-counting luminosity plots for the $2017 \sqrt{s}=13 \mathrm{TeV}$ high-luminosity running period, http://atlas.web.cern.ch/Atlas/GROUPS/PHYSICS/PLOTS/LUMI-2017-002/.

[4] ATLAS Collaboration, Performance of the ATLAS track reconstruction algorithms in dense environments in LHC Run 2, Eur. Phys. J. C (2017) 77: 673.

[5] ATLAS Collaboration, Modelling of Track Reconstruction Inside Jets with the 2016 ATLAS $\sqrt{s}=13$ TeV pp Dataset, ATL-PHYS-PUB-2017-016 (cdsweb.cern.ch/record/2275639).

[6] ATLAS Collaboration, $p_{T}$-weighted track reconstruction efficiency inside jets with 2016 data, https://atlas.web.cern.ch/Atlas/GROUPS/PHYSICS/PLOTS/IDTR-2016-019/.

[7] ATLAS Collaboration, Study of the mechanical stability of the ATLAS Insertable B-Layer, ATL-INDET-PUB-2015-001 (cdsweb.cern.ch/record/2022587).

[8] ATLAS Collaboration, Weak mode biases in final ID alignment for the 2016 pp dataset, https://atlas.web.cern.ch/Atlas/GROUPS/PHYSICS/PLOTS/IDTR-2017-005/.

[9] ATLAS Collaboration, Studies of radial distortions of the ATLAS Inner Detector, ATL-PHYS-PUB-2018-003 (cdsweb.cern.ch/record/2309785).

[10] ATLAS Collaboration, Measurements of $b$-jet tagging efficiency with the ATLAS detector using $t \bar{t}$ events at $\sqrt{s}=13 \mathrm{TeV}$, JHEP 1808 (2018) 089.

[11] ATLAS Collaboration, Calibration of light-flavour b-jet mistagging rates using ATLAS proton-proton collision data at $\sqrt{s}=13 \mathrm{TeV}$, ATLAS-CONF-2018-006 (cdsweb.cern.ch/record/2314418).

[12] ATLAS Collaboration, Measurement of b-tagging efficiency of c-jets in tī events using a likelihood approach with the ATLAS detector, ATLAS-CONF-2018-001 (cdsweb.cern.ch/record/2306649).

[13] ATLAS Collaboration, Optimisation and performance studies of the ATLAS b-tagging algorithms for the 2017-18 LHC run, ATL-PHYS-PUB-2017-013 (cdsweb.cern.ch/record/2273281). 